\title{
A molecular phylogeny of nuclear and mitochondrial sequences in Hymenolepis nana (Cestoda) supports the existence of a cryptic species
}

\author{
M. G. MACNISH ${ }^{1}$, U. M. MORGAN-RYAN ${ }^{1}$, P. T. MONIS ${ }^{2}$, J. M. BEHNKE \\ and R. C. A. THOMPSON ${ }^{1 *}$ \\ ${ }^{1}$ WHO Collaborating Centre for the Molecular Epidemiology of Parasitic Infections and Division of Veterinary and \\ Biomedical Sciences, Murdoch University, Murdoch, Western Australia, Australia 6150 \\ ${ }^{2}$ Microbiology Unit, Australian Water Quality Centre, South Australian Water Corporation, Hodgson Road, \\ Bolivar 5110, Australia \\ ${ }^{3}$ School of Life and Environmental Sciences, University of Nottingham, University Park, Nottingham NG7 2RD, UK
}

(Received 18 March 2002; revised 11 Fuly 2002; accepted 15 fuly 2002)

\section{SUMMARY}

Since isolates of Hymenolepis nana infecting humans and rodents are morphologically indistinguishable, the only way they can be reliably identified is by comparing the parasite in each host using molecular tools. In the current study, isolates of H. nana from rodent and human hosts from a broad geographical range were sequenced at the ribosomal first internal transcribed spacer (ITS1), the mitochondrial cytochrome $c$ oxidase subunit 1 (C01) gene and the nuclear paramyosin gene loci. $\dagger$ Twenty-three isolates of H. nana were sequenced at the ITS1 locus and this confirmed the existence of spacers which, although similar in length (approximately $646 \mathrm{bp}$ ), differed in their primary sequences which led to the separation of the isolates into 2 clusters when analysed phylogenetically. This sequence variation was not, however, related to the host of origin of the isolate, thus was not a marker of genetic distinction between $H$. nana from rodents and humans. Sequencing of a $444 \mathrm{bp}$ fragment of the mitochondrial cytochrome $c$ oxidase 1 gene (C01) in 9 isolates of $H$. nana from rodents and 6 from humans identified a phylogenetically supported genetic divergence of approximately $5 \%$ between some mouse and human isolates. This suggests that $H$. nana is a species complex, or 'cryptic' species (=morphologically identical yet genetically distinct). A small segment of the nuclear gene, paramyosin, (625 bp or $840 \mathrm{bp}$ ) was sequenced in 4 mouse and 3 human isolates of H. nana. However, this gene did not provide the level of heterogeneity required to distinguish between isolates from rodent and human hosts. From the results obtained from faster evolving genes, and the epidemiological evidence, we believe that the life-cycle of $H$. nana that exists in the north-west of Western Australia is likely to involve mainly 'human to human' transmission.

Key words: Hymenolepis nana, cryptic species, ribosomal ITS1, mitochondrial C01, paramyosin.

\section{INTRODUCTION}

The tapeworm Hymenolepis nana was first described as Taenia nana by Von Siebold in 1852 as a parasite found in humans. In 1906 Stiles described a morphologically identical parasite from a rodent host and named it Hymenolepis nana var. fraterna (see Joyeux, 1920 and Skrabin \& Matevosan, 1945 in Baer \& Tenora, 1970). Controversy over their status as a single or dual species and host specificity has existed ever since (Baer \& Tenora, 1970; Schantz, 1996). It is not entirely clear whether the species Hymenolepis

* Corresponding author: Murdoch University, South Street, Murdoch, WA 6150. Tel: +61 89360 2466. Fax: +6189310 4144. E-mail: andrew_t@central.murdoch. edu.au

$\dagger$ Nucleotide sequence data published here have been submitted to GenBank ${ }^{\mathrm{TM}}$ and are available under accession numbers AF461124 and AF461125 (18S-28S); AY121842 and AY121843 (cytochrome $c$ oxidase 1); AY1844 and AY121845 (paramyosin). nana and Hymenolepis fraterna are 2 distinct species, each highly host specific; whether they are 2 distinct species but capable of infecting both human and rodent hosts or whether they are simply the same species found in either host (see Brumpt, 1949 and Yamaguti, 1959 in Baer \& Tenora, 1970; Ferretti, Gabriele \& Palmas, 1981).

Further nomenclature difficulties are encountered with the re-classification of Hymenolepidids with armed rostella (hooks present) as Rodentolepis (Spasskii, 1954). Thus, H. nana (von Siebold, 1852) and $H$. fraterna (Stiles, 1906) are now classified as Rodentolepis nana and $R$. fraterna respectively by some taxonomists. Despite this revised nomenclature, the original confusion of speciation and host specificity remains to be solved. In a recent study, the oral inoculation of 51 samples of $H$. nana of human origin into specific pathogen-free hamsters, 4 mouse strains and 2 rat strains failed to establish infections (Macnish et al. 2002). Furthermore, inoculation of the same samples into thymus deficient- and cortisone 
Table 1. Source and geographical location of parasite material used in this study

(AI, Dr Akira Ito, Gifu University, Japan; MC, Dr Margherita Conchedda, Università degli Studi di Cagliari, Italy; MUPTR, Murdoch University Parasitology Teaching Resource; JB, Dr Jerzy Behnke, University of Nottingham, UK; GS, Dr Grant Singleton, CSIRO, NSW, Australia; MUPS, Murdoch University Parasite Survey.)

\begin{tabular}{|c|c|c|c|c|c|}
\hline $\begin{array}{l}\text { Known* or } \\
\text { presumed } \dagger \text { species }\end{array}$ & Host & Code & Sample type & Source & Geographical location \\
\hline H. nana* & Mouse & M1 & Adult worm & AI & Japan \\
\hline H. nana* & Mouse & $\mathrm{M} 2 \Delta$ & Cysticercoids & $\mathrm{MC}$ & Italy \\
\hline H. diminuta* & Rat & $\mathrm{Hd}$ & Adult worm & MUPTR & Perth, Western Australia \\
\hline H. citelli* & Hamster & Hc & Adult worm & $\mathrm{JB}$ & United Kingdom \\
\hline H. microstoma* & Mouse & $\mathrm{Hm}$ & Adult worm & JB & United Kingdom \\
\hline H. microstoma* & Mouse & M3, M4 & Adult worm & JB & Quinta de Sao Pedro, Portugal \\
\hline H. nana* & Mouse & M5, M6 & Adult worm & JB & Quinta de Sao Pedro, Portugal \\
\hline H. nana & Mouse & M7-M15 & Eggs in faeces & GS & Victoria, Australia \\
\hline H. nana & Human & H1-H12 & Eggs in faeces & MUPS & North-west Western Australia \\
\hline
\end{tabular}

* Morphologically identified adult worm (except M2 $\Delta=$ cysticercoids).

$\dagger$ Identified by egg morphology only.

acetate treated-mice, rats and hamsters also failed to establish infections, providing supportive evidence for the hypothesis that the species in mice may be non-infective to humans, and thus, represent a hostspecific 'strain' (Macnish et al. 2002).

The form/'strain' of $H$. nana present in Australia has never been identified with certainty. Furthermore, it is not well understood which form of transmission commonly occurs in Australian communities, whether the 'strain/species' present in the north-west of Western Australia is infective to human and rodent hosts, or whether humans harbour their own 'strain/species' of Hymenolepis. Since isolates of $H$. nana infecting humans and rodents are morphologically identical, the only way they can be reliably distinguished is by comparing the parasite in each host using molecular techniques.

To date, no comprehensive study of the molecular characteristics of $H$. nana isolates from humans or rodents has been carried out. In one study, sequences of the internal transcribed spacer 2 (ITS2) region of ribosomal DNA and partial sequences of the mitochondrial cytochrome $c$ oxidase subunit 1 (C01) gene were compared between an isolate of $H$. nana, collected from a laboratory mouse (Mus musculus) from Japan, and a laboratory golden hamster (Mesocricetus auratus) from Uruguay. No sequence differences were found in the ITS2 between both isolates and only 2 base differences were detected in the C01 locus (Okamoto et al. 1997).

Understanding the status of the 2 putative species $H$. nana and $H$. fraterna, and their host predilection, is of biological, epidemiological and taxonomic importance. Molecular characterization of these parasites, collected from both human and rodent hosts from a wide geographical distribution, will provide further evidence towards their identity. Understanding the genotypic characteristics of Hymenolepis isolates in different hosts will also help in determining host specificity and transmission patterns and thus allow a more appropriate approach to the control of infections in endemic communities. The aim of this study was, therefore, to sequence the ribosomal ITS1, mitochondrial C01 and part of the paramyosin gene of numerous Hymenolepis isolates collected from humans and mice from several geographically separated regions to ascertain whether any significant genetic differences existed between $H$. nana isolates from the two host types.

\section{MATERIALS AND METHODS}

\section{Collection of parasite material}

Sources of all parasites used in this study are listed in Table 1. A reference isolate of Hymenolepis nana was obtained from Dr Akira Ito, Gifu University, Japan. Approximately $2000 \mathrm{H}$. nana eggs were inoculated into 5-week-old male BALB/c mice. Adult worms were dissected from the small intestine approximately 14 days post-inoculation then washed repeatedly in phosphate-buffered saline (PBS) and stored at $-80{ }^{\circ} \mathrm{C}$ until DNA extraction. $H$. diminuta adult worms were obtained by dissection of infected 6-week-old male Wistar rats maintained by the Murdoch University Parasitology teaching resource. Adult worms of $H$. citelli (hamsters) and $H$. microstoma (mice) preserved in dimethyl sulphoxidesaturated $\mathrm{NaCl}$ were supplied by Dr Jerzy Behnke.

\section{Purification of DNA from adult worms and cysticercoids}

DNA was purified from $H$. nana, H. diminuta, $H$. microstoma and $H$. citelli using the QIAmp tissue purification kit (Qiagen, Hilden, Germany) with some minor modifications. Briefly, $10 \mu \mathrm{l}$ of glass milk matrix (Bio-Rad, California, USA) was substituted from the QIAmp spin columns as suggested by Morgan et al. (1998). DNA was eluted in $300 \mu$ of 
Tris-EDTA (TE) and $1 \mu \mathrm{l}$ of the diluted extract was added to the polymerase chain reaction (PCR) mix.

\section{Purification of DNA from human and mouse faeces}

DNA was purified from mouse faecal samples as previously described (Morgan et al. 1998). DNA was purified from human faecal samples using a method first described by Walsh, Metzger \& Higuchi (1991) and modified by Paxinos et al. (1997). Some further modifications were used in our laboratory. Briefly, a small plug of faecal material was suspended in $250 \mu \mathrm{l}$ of $10 \%$ Chelex $^{\circledR} 100$ (Bio-Rad, California, USA) in TE buffer, boiled for $7 \mathrm{~min}$ and vortexed vigorously. Samples were boiled again for $7 \mathrm{~min}$ then centrifuged at full speed for $5 \mathrm{~min}$. The supernatants were de-proteinized using ProCipitate ${ }^{\mathrm{TM}}$ (LigoChem Inc, USA), which is a non-hazardous alternative to phenol/chloroform. Briefly, the supernatant was mixed with an equal volume of ProCipitate ${ }^{\mathrm{TM}}$ and mixed gently for $5 \mathrm{~min}$ at room temperature. The samples were centrifuged at full speed for $5 \mathrm{~min}$. The supernatant was further concentrated using standard sodium acetate/ethanol precipitation then eluted in $50 \mu \mathrm{l}$ of TE. Usually $2 \cdot 5 \mu \mathrm{l}$ of template DNA was used for subsequent $\mathrm{PCR}$ reactions.

\section{Primer design for amplification of ITS1}

DNA sequences of $H$. nana and $H$. diminuta spanning the $3^{\prime}$ end of the $18 \mathrm{~S}$ rRNA gene, internal transcribed spacer 1 (ITS1), 5.8S, ITS2 and the 5' end of the $28 \mathrm{~S}$ rRNA gene $\left(\mathrm{GenBank}^{\mathrm{TM}}\right.$ accession numbers AF461124 and AF461125 respectively) were used to identify regions conserved between the 2 species of interest. Primers from these regions, designated F3 (5' GCGGAAGGATCATTACACGTTC 3') and R3 (5' GCTCGACTCTTCATCGATCCACG $3^{\prime}$ ) were designed using the software package Amplify 2.1 (Bill Engels, University of Wisconsin) to allow the amplification of the ITS1 regions of $H$. nana and $H$. diminuta.

\section{PCR amplification and sequencing of ITS1}

DNA was amplified in $67 \mathrm{~mm}$ Tris- $\mathrm{HCl}(\mathrm{pH} 8 \cdot 8)$, $16.6 \mathrm{~mm}\left(\mathrm{NH}_{4}\right)_{2} \mathrm{SO}_{4}, 2 \mathrm{~mm} \mathrm{MgCl}_{2}, 0 \cdot 5$ unit of Tth plus (Fisher Biotech, Perth, Australia), $200 \mu \mathrm{M}$ of each dNTP and 12.5 pmoles of each primer. Reactions were performed on a PE 2400 (Perkin Elmer, Foster City, California) thermal cycler. Samples were heated to $94{ }^{\circ} \mathrm{C}$ for $2 \mathrm{~min}, 63{ }^{\circ} \mathrm{C}$ for $2 \mathrm{~min}, 72{ }^{\circ} \mathrm{C}$ for $1 \mathrm{~min}$, followed by 50 cycles of $94^{\circ} \mathrm{C}$ for $20 \mathrm{sec}, 63{ }^{\circ} \mathrm{C}$ for $20 \mathrm{sec}, 72^{\circ} \mathrm{C}$ for $45 \mathrm{sec}$ and a final cycle of $72{ }^{\circ} \mathrm{C}$ for 7 min. Usually $0 \cdot 5$ unit of Taq Extender (Strategene, USA) was added to the PCR mix to improve amplification efficiency.

Amplification products were purified using the QIAquick-spin PCR purification kit (Qiagen,
Germany) and sequenced in both directions with F3 and R3 primers using an ABI Prism ${ }^{\mathrm{TM}}$ Dye Terminator Cycle Sequencing Ready Reaction kit (Applied Biosystems, Foster City, California) according to manufacturer's instructions with some modifications. Briefly, the reagent volumes were halved and the annealing temperature was raised to $60{ }^{\circ} \mathrm{C}$. In some instances, $2 \mu \mathrm{l}$ of Half'TERM (Genpak Inc, Stony Brook, New York) was substituted for $2 \mu \mathrm{l}$ of dye terminator mix as this reduced the cost of the reaction without compromising the quality of the sequence. The sequences were analysed using SeqEd v1.0.3 (Applied Biosystems).

When direct sequencing of the PCR product yielded poor results PCR products were cloned into a $\mathrm{pCR}^{\circledR} 2.1 \mathrm{~T}$-vector (Invitrogen, USA) and transformants were screened by PCR. Plasmid DNA was purified from overnight cultures using the FlexiPrep ${ }^{\circledR}$ kit (Pharmacia Biotech Inc, USA). At least 3 positive clones were sequenced in both directions using universal M13 primers.

\section{PCR amplification and sequencing of mitochondrial C01}

A segment of the mitochondrial cytochrome $c$ oxidase subunit 1 (C01) was amplified using primers and conditions described by Okamoto et al. (1997) with a single modification which was to increase the annealing temperature from $42{ }^{\circ} \mathrm{C}$ to $55^{\circ} \mathrm{C}$. This modification was required to prevent non-specific amplification because problems were encountered with non-specific primer binding from DNA extracted from faecal samples.

When direct sequencing of the C01 fragment yielded poor results the PCR product was cloned in the manner described for the ITS1 fragments and sequenced using universal M13 forward and reverse primers.

\section{Primer design, PCR amplification and sequencing of nuclear paramyosin (pmy)}

A degenerate forward primer, designated Pmy-F (5' AAYCAYYTVAGTCCGAGATGGAAC 3') and located approximately $1550 \mathrm{bp}$ downstream of the $5^{\prime}$ end of pmy was designed using available sequences of the closely related species Echinococcus granulosus (GenBank $^{\mathrm{TM}}$ accession number Z21787), Taenia solium (L13723), Schistosoma japonicum (AF113971 and U11825) and S. mansoni (M35499). A degenerate reverse primer, designated Pmy-R (5' ACCATACGRCGACCYTCACGDGTAGC 3') was a modified version of a primer designed by Laclette et al. (1991). Amplification of DNA extracted from whole worms was achieved using these primers. However, some difficulties were encountered with the amplification of pmy from DNA extracted from eggs in faeces. A nested PCR approach was used instead. Two new sets 
of primers, Ext-F (5' AGAAAGAGCACCACTCGCAC $3^{\prime}$ ) were located just $3^{\prime}$ of the Pmy-F primer. A conserved new external reverse primer, Ext-R ( $5^{\prime}$ GACAGTAATCTCACGGATCTC 3') was located just 3' of the Pmy-R primer. The external set of primers, Ext-F and Ext-R amplified a 700 bp product. The internal set of primers, Int-F (5' ATTTCTGAGATGGAGGTCAGATTTAAG $3^{\prime}$ ) and Int R (5'TTTGCGAAGAGTTTCAGCACGCTTG 3'), amplified a 625 bp product. DNA was amplified in $25 \mu 1$ vol. reactions as for the ITS 1 and C01 loci, except that $25 \mathrm{pmol}$ of each primer was used and the $\mathrm{MgCl}_{2}$ concentration was increased to $3 \mathrm{mM}$.

For the primary PCR reaction samples were heated to $94{ }^{\circ} \mathrm{C}$ for $3 \mathrm{~min}$ followed by 50 cycles of $94{ }^{\circ} \mathrm{C}$ for $30 \mathrm{sec}, 58^{\circ} \mathrm{C}$ for $20 \mathrm{sec}, 72{ }^{\circ} \mathrm{C}$ for $45 \mathrm{sec}$ and a final cycle of $72{ }^{\circ} \mathrm{C}$ for $7 \mathrm{~min}$. One $\mu \mathrm{l}$ of the primary PCR reaction was used as a template for the secondary nested PCR reaction. Samples were heated to $94{ }^{\circ} \mathrm{C}$ for $1 \mathrm{~min}$, followed by 50 cycles of $94^{\circ} \mathrm{C}$ for $3 \mathrm{sec}$, $70{ }^{\circ} \mathrm{C}$ for $20 \mathrm{sec}, 72{ }^{\circ} \mathrm{C}$ for $45 \mathrm{sec}$ and a final cycle of $72{ }^{\circ} \mathrm{C}$ for $7 \mathrm{~min}$.

\section{Phylogenetic analyses}

Nucleotide sequences were aligned using Clustal X (Thompson et al. 1997). Distance-based and parsimony analyses were performed using PAUP* (Swofford, D. L. 1999. PAUP*. Phylogenetic Analysis Using Parsimony (*and Other Methods). Version 4.0b2 Sinauer Associates, Sunderland, Massachusetts). Maximum Likelihood analyses were performed using PUZZLE (version 4.1, (Strimmer \& von Haeseler, 1996)). Distance-based analyses were conducted using Tamura-Nei distance estimates and trees were constructed using the Neighbour-Joining algorithm. Parsimony analyses were conducted using either the branch and bound or heuristic search methods. Bootstrap analyses were conducted using 1000 replicates. Trees were drawn using the TreeView program (Page, 1996).

\section{RESULTS}

\section{Sequence analysis of ITS1}

The ITS1 region was sequenced from 23 isolates of H. nana (11 human, 12 mouse). Amplified ITS1 was cloned for 11 isolates and between 2 and 6 clones were sequenced for each isolate resulting in a total of 37 clones being analysed. Amplicons from the remaining isolates (5 human, 7 mouse) were sequenced directly. Sequence analysis determined that the PCR products from $H$. nana included $22 \mathrm{bp}$ of the $3^{\prime}$ end of the $18 \mathrm{~S}, 571 \mathrm{bp}$ of the ITS1 and 53 bases of the $5^{\prime}$ end of the $5 \cdot 8 \mathrm{~S}$. Although 23 isolates were analysed, only 6 distinct sequence types were identified. Of these 6 sequences, 13 isolates (H7, M7, M1, M12, H10, M2, H8, M9c1, H11c3, H11c1, M11c3, H2c3) possessed one sequence and 5 (M5, M6, H4c2, $\mathrm{H} 4 \mathrm{c} 1, \mathrm{H} 6 \mathrm{c} 2)$ possessed another. The 2 predominant sequences were obtained from both cloned and directly sequenced amplified DNA's.

\section{Phylogenetic analysis of ITS1}

Analysis of ITS1 nucleotide sequences was conducted using $H$. microstoma as an outgroup. Due to the uncertainties regarding the basis for the high levels of variability among cloned sequences from individual isolates of $H$. nana, each clone was included in the final phylogenetic analysis (Fig. 1). Analysis of ITS1 sequences provided confirmation that isolates M3, M4 and M15c4 are H. microstoma. Distance-based and Maximum Likelihood (ML) analyses identified 2 main clusters of isolates (Clusters 1 and 2, Fig. 1). Cluster 1, containing M5, M6, H3c1-c4, H4c1-c2, H6c1-c3, was supported by bootstrap analysis ( $89 \%$ ) (Fig. 1). The topology of the tree for the remaining isolates of $H$. nana received poor bootstrap support. Parsimony analysis of these data was not possible due to the large number of trees with the same length generated. The most substantial sequence variation was seen between $H$. nana sequences in Cluster 1 versus those in Cluster 2. Two directly sequenced isolates from Portugal (M5, M6) shared identical sequences with cloned isolates within Cluster 1. Some variation was also seen between the isolates within Cluster 2 itself; however, this was usually low $(98 \cdot 8-99 \cdot 4 \%)$.

\section{Sequence analysis of $\mathrm{C} 01$}

Sequence analysis determined that the PCR product obtained by amplification with primers pr-a and pr-b was $444 \mathrm{bp}$ for $H$. nana (PCR results not shown). Direct sequences were obtained for $H$. diminuta (411 bp), H. microstoma (429 bp) and $H$. citelli (425 bp). At the genus level, H. nana was $85,81 \cdot 3$ and $81 \cdot 7 \%$ genetically similar to the 3 other Hymenolepidids $H$. microstoma, $H$. diminuta and $H$. citelli respectively (data not shown). Intra-specific variation was not detected between the human isolates of $H$. nana and was very low $(99 \cdot 5-100 \%)$ between the mouse isolates from Australia (M9, M11, M12, M13, M14), Japan (M1) and Italy (M2). However, extensive intra-specific variation was found between the 2 Portugese mouse isolates, M5 and M6, and the remaining mouse isolates (M1, M2, M9, M11, M12, M13, M14) ranging from 95.0 to $96.0 \%$. Similarly, high levels of intra-specific variation between the human isolates and the 2 rodents isolates, M5, M6, were observed $(96 \cdot 1 \%)$. Variation within an individual isolate, ascertained by sequencing 3 clones, was only observed for the $H$. nana isolate M6 and was low (98.8\%). The remaining isolates of $H$. nana and other Hymenolepis species were sequenced directly and no 


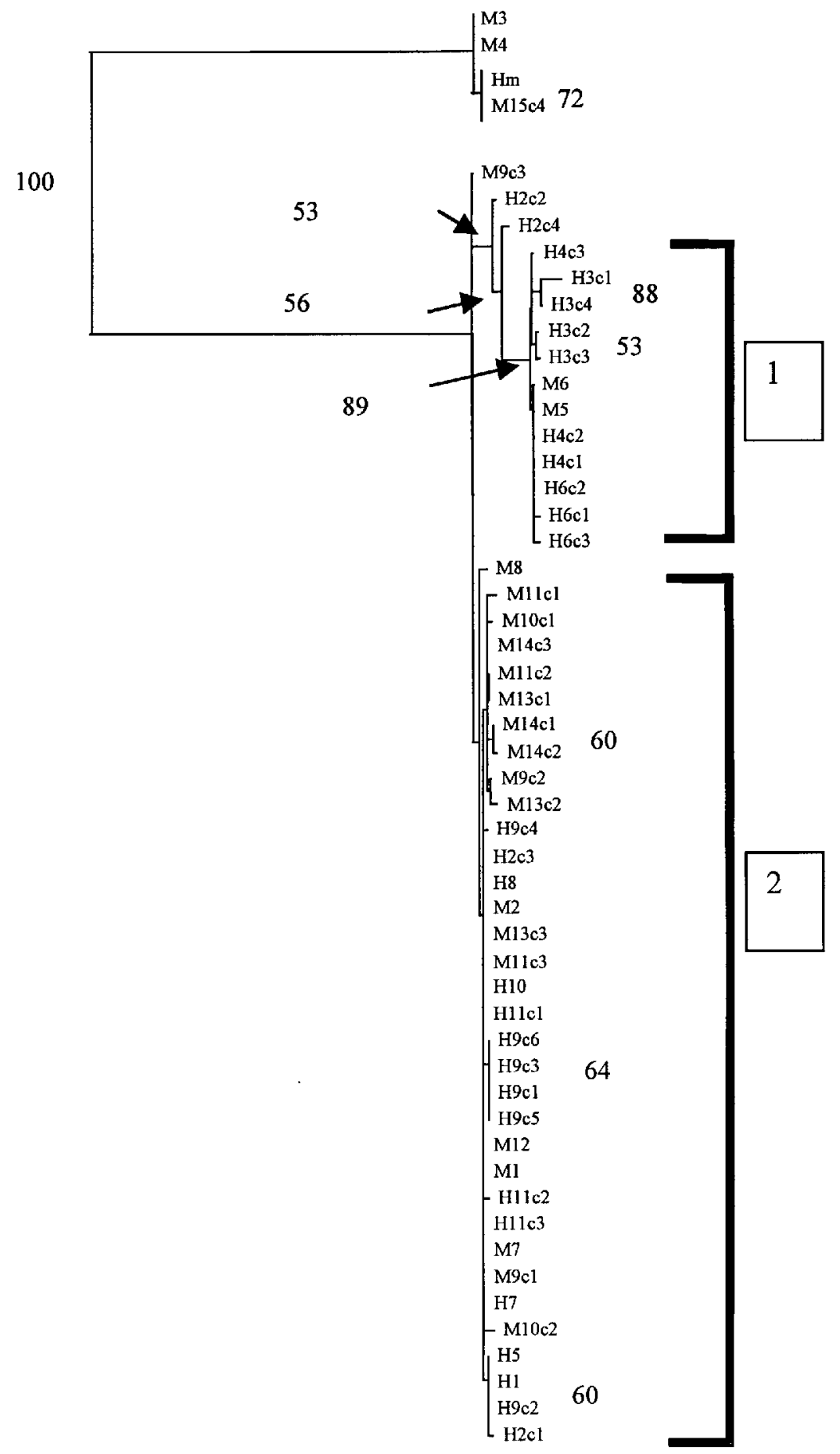

Fig. 1. Phylogram of distance-based analyses generated from the sequence of the ribosomal internal transcribed spacer 1 (ITS1) gene region from human (H) and mouse (M) isolates of Hymenolepis nana and from H. microstoma (Hm).

polymorphisms were found in the region sequenced for any species.

\section{Phylogenetic analysis of mitochondrial C01}

Analysis of C01 nucleotide sequences was conducted using $H$. diminuta and $H$. citelli as outgroups. Parsimony, distance-based and ML analyses produced trees with similar topology (Fig. 2). The rodent isolates M3, M4 and M15 were identified as $H$. microstoma. Isolate M15 was placed into the same clade as the H. microstoma reference sequence but this was poorly supported by bootstrap analysis. The isolates of $H$. nana were divided into 2 clades, one containing the mouse isolates M5 and M6 and the other containing the remaining human- and mousederived isolates of $H$. nana. The topology within the latter clade suggests a division correlating with host 


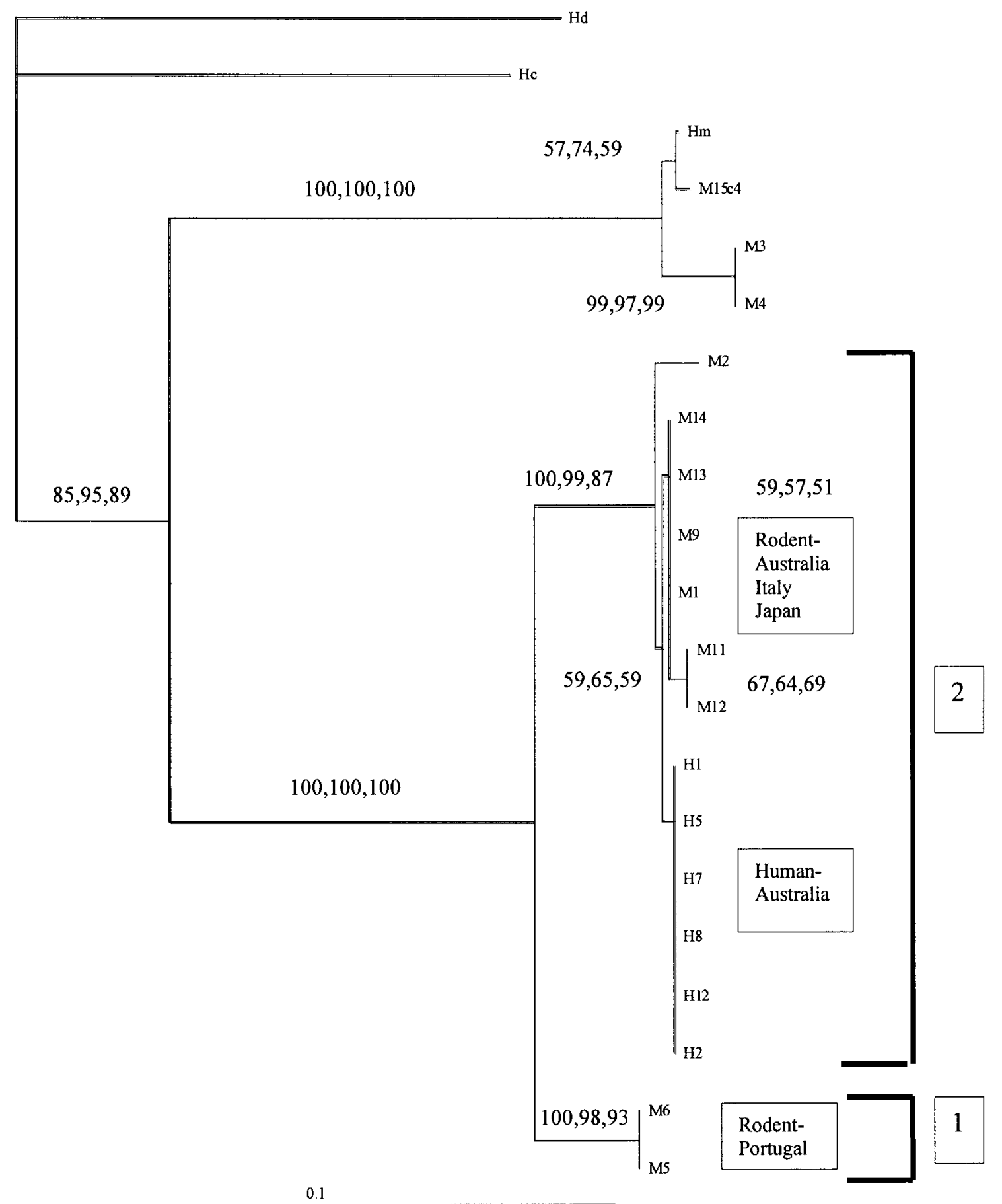

Fig. 2. Phylogram of distance-based analyses generated from the sequences of the mitochondrial cytochrome $c$ oxidase subunit 1 (C01) gene region from human $(\mathrm{H})$ and mouse (M) isolates of Hymenolepis nana and from H. diminuta (Hd), H. microstoma $(\mathrm{Hm})$, H. citelli $(\mathrm{Hc})$.

origin, with isolates from the same host species clustering with each other. However, this topology was not supported by bootstrap analysis.

Sequence analysis of paramyosin

A PCR product of approximately $840 \mathrm{bp}$ was obtained from the H. nana isolates M1, M2, M5, M6, $H$. microstoma, M3, H. diminuta and $H$. citelli using the primers Pmy-F and Pmy-R (results not shown). Direct sequencing of approximately $840 \mathrm{bp} \mathrm{PCR}$ product, using the primers Pmy-F and Pmy-R, was achieved with the $H$. nana isolates M1, M2, M5, M6, the reference isolate of H. microstoma, and the field isolate $\mathrm{M} 3, H$. diminuta and $H$. citelli. Unambiguous sequence of $782 \mathrm{bp}, 775 \mathrm{bp}, 796 \mathrm{bp}$, $788 \mathrm{bp}$ was obtained for H. nana, H. microstoma, $H$. diminuta and $H$. citelli respectively. Direct 


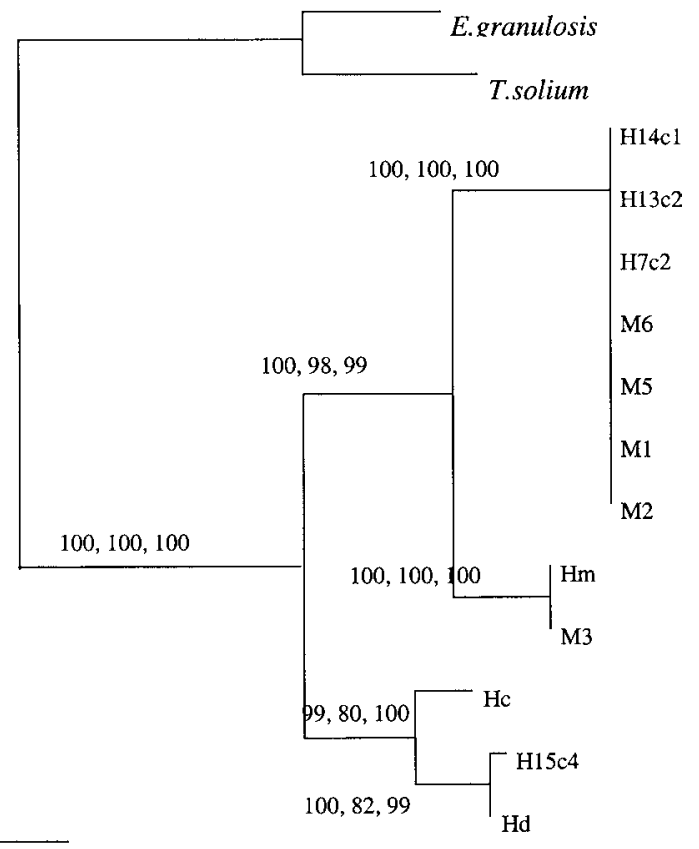

0.1 nt substitutions / site

Fig. 3. Phylogram of distance-based analyses generated from the sequences of the paramyosin gene region from human $(\mathrm{H})$ and mouse $(\mathrm{M})$ isolates of Hymenolepis nana and from $H$. diminuta $(\mathrm{Hd}), H$. microstoma $(\mathrm{Hm}), H$. citelli (Hc). The cestodes Echinococcus granulosis and Taenia solium were used as outgroups (GenBank ${ }^{\text {TM }}$ accession numbers Z21787 and L13723 respectively).

sequencing of the PCR product obtained using the nested PCR primers of H. nana isolates H7, $\mathrm{H} 13, \mathrm{H} 14$ and $H$. diminuta isolate $\mathrm{H} 15$ yielded poor results therefore the PCR products were cloned prior to sequencing. Sequence analysis of this PCR product confirmed the size of the PCR product was $625 \mathrm{bp}$ which corresponded with the predicted fragment size using the secondary primers, Int-F and Int-R. Intra- and inter-specific variation between and within isolates of $H$. nana was not detected.

\section{Phylogenetic analysis of paramyosin}

Parsimony, distance and maximum likelihood (heuristic, quartet puzzling) analyses produced trees with the same topology (Fig. 3). Isolates of H. nana (human and mouse) possessed identical paramyosin nucleotide sequences and were placed into a single clade. H. microstoma was placed as the closest relative of H. nana. Hymenolepis diminuta and $H$. citelli were placed into the same clade and formed a sister group to $H$. nana/H. microstoma. The human isolate $\mathrm{H} 15 \mathrm{c} 4$ was identified as $H$. diminuta based on sequence similarity and phylogeny. All of the nodes of the tree were very highly supported by bootstrap analysis using the distance-based and ML methods (99-100\%). Bootstrap analysis using parsimony found high support (98-100\%) for the grouping of the $H$. nana and $H$. microstoma but lower support (80-82\%) for the grouping of $H$. diminuta with $H$. citelli. The monophyly of Hymenolepis was highly supported $(100 \%)$ with respect to the outgroups used in the study.

\section{DISCUSSION}

A total of 23 isolates (human and mouse) of H. nana, representing a wide geographical distribution (Australia, Japan, Italy, Portugal) were characterized, by sequencing, at the ribosomal ITS1 locus. Of these, 14 isolates were also characterized at the mitochondrial C01 locus and 7 at the paramyosin locus. More isolates were unable to be sequenced at the $\mathrm{C} 01$ and paramyosin loci due to insufficient material. Phylogenetic analysis of the ITS1 region of these isolates identified 2 clusters whose composition did not correlate with host (mouse or human) or geographical origin of the isolates. Variation was found both between and within isolates of H. nana. The basis of the variation within a single isolate was not determined but could be due to either variation between eggs within a sample or variation between ITS repeats within an individual egg. Detailed insight into 'strain resolution' between the mouse and human isolates was not possible using this locus due to the high levels of polymorphism (Vogler \& DeSalle, 1994; Sorensen, Curtis \& Mindhella, 1998; Jobst, King \& Hemleben, 1998).

Sequencing of the mitochondrial cytochrome $c$ oxidase 1 gene (C01) in a number of isolates of $H$. nana from rodents and humans identified a phylogenetically supported genetic divergence between 
some mouse isolates in comparison with isolates of $H$. nana from humans. This provided evidence that the mitochondrial $\mathrm{C} 01$ gene was useful for identifying genetic divergences in $H$. nana that were not resolvable using nuclear loci. The difficulties in amplifying mitochondrial genes from egg DNA meant that important information was lost for some isolates, such as $\mathrm{H} 2, \mathrm{H} 3, \mathrm{H} 4$ and $\mathrm{H} 6$, at the $\mathrm{C} 01$ locus. Genetic characterization of these particular isolates, at the C01 locus, would be invaluable for a direct comparison to be made between all the isolates characterized in this study.

In the current study, the placement of the 2 Portugese isolates (M5, M6) into a separate clade, as a result of $5.0 \%$ genetic divergence at the C01 locus that is well supported by bootstrap analysis, is highly suggestive of the existence of 'cryptic species' of H. nana (=genetically distinct yet morphologically identical). Given the geographical isolation of Portugal from Australia it is possible that distinct genotypes would evolve over time in this region. In addition, the separation of all the human isolates into a group within Cluster 2, whilst not supported by high bootstrap values, is well supported by biological data obtained in a previous study (Macnish et al. 2002) suggesting that a barrier to gene flow may be occurring in the Australian populations of H. nana. This may be due to environmental and/or ecological pressures caused by the documented absence of domestic mice species in close proximity to human dwellings, combined with the susceptibility or resistance of the host (genetic factors, host immunity, host diet). In addition, selection pressure by the host and/ or the parasite may contribute to the co-evolution of particular host-parasite relationships.

The region of the $P m y$ gene characterized in this study yielded phylogenetically informative data for the resolution of the relationships between $H$. nana, $H$. microstoma, $H$. diminuta and $H$. citelli that corresponded with the relationships found using 2 other genetic loci; the nuclear ribosomal ITS1 and the mitochondrial C01. However, the gene was too conserved to allow differentiation of $H$. nana isolates from different hosts.

Currently, the 'yardstick' for delineating 'species/ strains' on the basis of genetic differences is unresolved in the literature and remains contentious (Morgan \& Blair, 1995; Haag et al. 1997; Sorensen et al. 1998; Blouin et al. 1998). Although some have proposed that if 'within-species' variation at particular genetic loci is low this supports the existence of a species (e.g. see Hung et al. 1999). Others suggest that caution is required in interpreting the genetic data in the absence of any supportive biological data (Thompson \& Lymbery, 1990; Blouin et al. 1998; Thompson, Constantine \& Morgan, 1998; Sorensen et al. 1998; Tibayrenc, 1998). The inference, based on sequence data from the CO1 locus, that H. nana is a species complex/cryptic species that differs in its host range is consistent with biological data reported earlier for these isolates (Macnish et al. 2002). From an epidemiological viewpoint this provides highly useful information that helps identify whether transmission is likely to be occurring between rodent and human hosts.

In light of the data presented here, and the epidemiological evidence (Macnish et al. 2002) we believe that the life-cycle of $H$. nana that exists in the north-west of Western Australia is likely to involve mainly 'human to human' transmission. In other communities worldwide, where poor hygiene practices help to promote the direct transmission of parasite species such as Hymenolepis, it is not clear whether mice act as reservoirs of strains of $H$. nana that are transmissible to humans. From the results of this study it would appear that, where rodent hosts are minimal or absent, the potential exists for the route of transmission to become mainly direct ('human to human') in those communities also.

It is now recommended to sequence faster evolving loci to interpret the relationship between human and mouse isolates with more clarity. Recently, the nicotinamide adenine dinucleotide dehydrogenase subunit 2 and 4 (ND2 and ND4 respectively) genes were characterized in 8 cestode species, including $H$. nana (Nakao et al. 2000). Further characterization of the ND4 mitochondrial gene in human and rodent isolates of $H$. nana is facilitated by this recent research and is recommended for future characterization of this parasite.

The authors would again like to thank Dr Akira Ito, Gifu University, Japan, and Dr Margherita Conchedda, Cagliari, Italy for their generous gifts of $H$. nana isolates. Funding provided by the Australian Federation of University Women (WA) Inc. and the National Health and Medical Research Centre (NHMRC) is also gratefully acknowledged.

\section{REFERENCES}

BAER, J. G. \& TENORA, F. (1970). Some species of Hymenolepis (Cestoidea) from rodents and from primates. Acta Scientiarum Naturalium Academiae Scientiarum Bohemoslovacae Brno 4, 1-32.

Blouin, M. S., Yowell, C. A., COURTNey, C. H. \& DAME, J. B. (1998). Substitution bias, rapid saturation, and the use of mtDNA for nematode systematics. Molecular Biology and Evolution 15, 1719-1727.

FERretTi, G., GABriele, F. \& PAlmas, C. (1981). Development of human and mouse strain of Hymenolepis nana in mice. International Fournal for Parasitology 11, 425-430.

haAG, K. L., ZAHA, A., ARAujo, A. M. \& GotTstein, B. (1997).

Reduced genetic variability within coding and non-coding regions of the Echinococcus multilocularis genome. Parasitology 115, 521-529.

HUNG, G. C., ChIlTON, N. B., BEveridge, I., ZHU, X. Q., Lichtenfels, J. R. \& GASSER, R. B. (1999). Molecular evidence for cryptic species within Cylicostephanus 
minutus (Nematoda: Strongylidae). International Fournal for Parasitology 29, 285-291.

JOBST, J., KING, K. \& HEMLEBEN, v. (1998). Molecular evolution of the internal transcribed spacers (ITS1 and ITS2) and phylogenetic relationships among species of the family Cucurbitaceae. Molecular Phylogenetics and Evolution 9, 204-219.

LACLETTE, J.P., LANDA, A., ARCOS, L., Willms, K., DAVIS, A. E. \& Shoemaker, C. B. (1991). Paramyosin is the Schistosoma mansoni (Trematoda) homologue of antigen B from Taenia solium (Cestoda). Molecular and Biochemical Parasitology 44, 287-296.

MACNISH, M. G., MORGAN, U. M., BEHNKE, J. M. \& THOMPSON, R. C. A. (2002). Failure to infect laboratory rodents with humans isolates of Rodentolepis (=Hymenolepis) nana. Fournal of Helminthology 76, 37-43.

MORGAN, J.A. T. \& BLAIR, D. (1995). Nuclear rDNA ITS sequence variation in the trematode genus Echinostoma: an aid to establishing relationships within the 37-collar-spine group. Parasitology 111, 609-615.

MORGAN, U. M., PALlant, L., DWyer, B. W., FORBES, D. A., RICH, G. \& THOMPSON, R. C. A. (1998). Comparison of PCR and microscopy for detection of Cryptosporidium parvum in human fecal specimens: Clinical trial. Fournal of Clinical Microbiology 36, 995-998.

NAKAO, M., SAKo, Y., YOKOYAMA, N., FUKUnAGa, M. \& ITO, A. (2000). Mitochondrial genetic code in cestodes. Molecular and Biochemical Parasitology 111, 415-424.

okamoto, M., agatsuma, T., Kurosawa, T. \& ito, A. (1997). Phylogenetic relationships of three hymenolepidid species inferred from nuclear ribosomal and mitochondrial DNA sequences. Parasitology 115, 661-666.

PAGE, R. D. M. (1996). TREEVIEW : an application to display phylogenetic trees on personal computers. Computer Applications in the Biosciences: CABIOS 12, 357-358.
PAXinOS, E., McINTOSH, C., RAlls, K. \& Fleischer, R.

(1997). A noninvasive method for distinguishing among canid species: amplification and enzyme restriction of DNA from dung. Molecular Ecology 6, 483-486. schantz, P. M. (1996). Tapeworms (Cestodiasis). Gastroenterology Clinics of North America 3, 637-653. SORENSEN, R. E., CURTIS, J. \& MindHELla, D. J. (1998). Intraspecific variation in the rDNA ITS loci of 37-collar-spined Echinostomes from North America: Implications for sequence-based diagnoses and phylogenetics. Fournal of Parasitology 84, 992-997. STRIMMER, K. \& VON HAESELER, A. (1996). Quartet puzzling: a quartet maximum likelihood method for reconstructing tree topologies. Molecular Biology and Evolution 13, 964-969.

Thompson, J. D., Gibson, T. J., Plewniak, F., JEAnmougin, F. \& HIGGINS, D. G. (1997). The Clustal X windows interface: flexible strategies for multiple sequence alignment aided by quality analysis tools. Nucleic Acids Research 25, 4876-4882.

Thompson, R. C. A., CONSTANTINe, C. C. \& MORGan, U. M. (1998). Overview and significance of molecular methods: what role for molecular epidemiology? Parasitology 117, S161-S175.

THOMPSON, R. C. A. \& LYMBeRY, A. J. (1990). Intraspecific variation in parasites - What is a strain? Parasitology Today 6, 345-348.

Tibayrenc, M. (1998). Genetic epidemiology of parasitic protozoa and other infectious agents: the need for an integrated approach. International fournal for Parasitology 28, 85-104.

VOGLER, A.P. \& DeSALle, R. (1994). Evolution and phylogenetic information content of the ITS-1 region in the tiger beetle Cicindela dorsalis. Molecular Biology and Evolution 11, 393-405.

Walsh, P. S., Metzger, D. A. \& Higuchi, R. (1991). Chelex 100 as a medium for simple extraction of DNA for PCR-bases typing from forensic material. BioTechniques 10, 506-513. 